doi: $10.2306 /$ scienceasia1513-1874.2013.39S.025

\title{
Optimal pursuit time for a differential game in the Hilbert space $l_{2}$
}

\author{
Gafurjan Ibragimov \\ Department of Mathematics, Faculty of Science, \& Institute for Mathematical Research, \\ Universiti Putra Malaysia, 43400, Serdang, Selangor, Malaysia \\ e-mail: gafur@science.upm.edu.my
}

Received 7 Jan 2013

Accepted 5 Apr 2013

\begin{abstract}
We consider a two-person zero-sum pursuit-evasion differential game in the Hilbert space $l_{2}$. The control functions of the players are subject to integral constraints. It is assumed that the control resource of the pursuer is greater than that of the evader. The pursuer tries to force the state of the system towards the origin of the space $l_{2}$, and the evader tries to avoid this. We give a solution to the optimal pursuit problem for the differential game. More precisely, we obtain an equation for the optimal pursuit time and construct optimal strategies for the players in an explicit form. To prove the main result we solve a time-optimal control problem.
\end{abstract}

KEYWORDS: pursuer, evader, optimal control, optimal strategy

\section{INTRODUCTION}

The study of two person zero-sum differential games was initiated by Isaacs ${ }^{1}$. Since then much work with various approaches has been done in developing the theory of differential games described by ordinary differential equations.

Control and differential game problems for systems described by partial differential equations are also of increasing interest ${ }^{4-10}$. In Refs. 4-9, such problems for the equations described by parabolic and hyperbolic equations were studied. By using the decomposition method these problems can be reduced to ones described by infinite systems of ordinary differential equations $6,8,9$

$$
\dot{z}_{k}+\lambda_{k} z_{k}=w_{k}, k=1,2, \ldots,
$$

where $w_{k}, k=1,2, \ldots$ are control parameters, $z_{k}$, $w_{k} \in R^{1}$, and $\lambda_{k}>0$ are eigenvalues of the elliptic operator defined by

$$
A=-\sum_{i, j=1}^{n} \frac{\partial}{\partial x_{i}}\left(a_{i j}(x) \frac{\partial}{\partial x_{j}}\right) .
$$

Hence there is a significant relationship between the control problems described by partial differential equations and those described by an infinite system of ODEs. The latter is of independent interest and can be investigated within one theoretical framework. Ref. 11 relates to such games described by an infinite system of ODEs and an evasion differential game with integral constraints was studied.
This paper is concerned with the so-called twoperson zero-sum pursuit-evasion differential game in the Hilbert space $l_{2}$ with integral constraints on the control functions of players. We investigate the differential game problem associated with the following infinite system of differential equations

$$
\begin{gathered}
\dot{x}_{k}=-\alpha_{k} x_{k}-\beta_{k} y_{k}+u_{1 k}-v_{1 k}, \\
\dot{y}_{k}=\beta_{k} x_{k}-\alpha_{k} y_{k}+u_{2 k}-v_{2 k}, \\
x_{k}(0)=x_{k 0}, \quad y_{k}(0)=y_{k 0},
\end{gathered}
$$

$k=1,2, \ldots$, in $l_{2}$, where $\alpha_{k}, \beta_{k}$ are real numbers, $\alpha_{k} \geqslant 0, x_{0}=\left(x_{10}, x_{20}, \ldots\right) \in l_{2}, y_{0}=$ $\left(y_{10}, y_{20}, \ldots\right) \in l_{2}, u=\left(u_{11}, u_{12}, u_{21}, u_{22}, \ldots\right)$ and $v=\left(v_{11}, v_{12}, v_{21}, v_{22}, \ldots\right)$ are control parameters of the pursuer and evader, respectively. The pursuer tries to force the state towards the origin of the space $l_{2}$ against any action of the evader who exactly tries to avoid this. We give a solution to the optimal pursuit problem for the differential game.

Note that if we substitute $\lambda_{k}=\alpha_{k}-\mathrm{i} \beta_{k}, z_{k}=$ $x_{k}+\mathrm{i} y_{k}, u_{k}=u_{1 k}+\mathrm{i} u_{2 k}, v_{k}=v_{1 k}+\mathrm{i} v_{2 k}, k=$ $1,2, \ldots$, into the system

$$
\dot{z}_{k}+\lambda_{k} z_{k}=u_{k}-v_{k}, k=1,2, \ldots
$$

then we obtain the system (1). Hence this work is a complement to Refs. 8, 9 .

\section{STATEMENT OF THE PROBLEM}

Let $\rho_{0}, \rho$ and $\sigma$ be positive numbers. 
Definition 1 A function

$$
\begin{array}{r}
w(\cdot)=\left(w_{11}(\cdot), w_{21}(\cdot), w_{12}(\cdot), w_{22}(\cdot), \ldots\right), \\
w:[0, T] \rightarrow l_{2},
\end{array}
$$

with measurable coordinates $w_{1 k}(t), w_{2 k}(t), 0 \leqslant t \leqslant$ $T, k=1,2, \ldots$, subject to the condition

$$
\sum_{k=1}^{\infty} \int_{0}^{T} w_{1 k}^{2}(s)+w_{2 k}^{2}(s) \mathrm{d} s \leqslant \rho_{0}^{2}
$$

is referred to as the admissible control, where $T>0$ is a sufficiently large fixed number.

We denote the set of all admissible controls by $S\left(\rho_{0}\right)$.

Definition 2 A function $u(\cdot) \in S(\rho)(v(\cdot) \in S(\sigma))$ is referred to as the admissible control of the pursuer (evader).

Definition 3 A function

$u(t, v)=\left(u_{1}(t, v), u_{2}(t, v), \ldots\right), u:[0, T] \times l_{2} \rightarrow l_{2}$,

with 2-d coordinates $u_{k}=\left(u_{1 k}, u_{2 k}\right)$ of the form

$$
\begin{gathered}
u_{k}(t, v)=v_{k}(t)+\omega_{k}(t), \\
\omega_{k}=\left(\omega_{k 1}, \omega_{k 2}\right), \quad v_{k}=\left(v_{1 k}, v_{2 k}\right), \\
\omega(\cdot)=\left(\omega_{1}(\cdot), \omega_{2}(\cdot), \ldots\right) \in S(\rho-\sigma),
\end{gathered}
$$

subject to the condition (admissibility)

$$
\sum_{k=1}^{\infty} \int_{0}^{T}\left|u_{k}(t, v(t))\right|^{2} \mathrm{~d} t \leqslant \rho^{2} \text { for any } v(\cdot) \in S(\sigma),
$$

is called a strategy of the pursuer.

$$
\begin{aligned}
\text { Let } & \\
z(t) & =\left(z_{1}(t), z_{2}(t), \ldots\right) \\
& =\left(x_{1}(t), y_{1}(t), x_{2}(t), y_{2}(t), \ldots\right), \\
\|z\| & =\left(\sum_{k=1}^{\infty}\left(x_{k}^{2}+y_{k}^{2}\right)\right)^{1 / 2}, \\
z_{k}(t) & =\left(x_{k}(t), y_{k}(t)\right),\left|z_{k}\right|=\sqrt{x_{k}^{2}+y_{k}^{2}}, \\
z_{0} & =\left(z_{10}, z_{20}, \ldots\right)=\left(x_{10}, y_{10}, x_{20}, y_{20}, \ldots\right), \\
\left\|z_{0}\right\| & =\left(\sum_{k=1}^{\infty}\left(x_{k 0}^{2}+y_{k 0}^{2}\right)\right)^{1 / 2} .
\end{aligned}
$$

Definition 4 If there exists a strategy $u(\cdot)$ of the pursuer such that for any admissible control of the evader the equality $z(\tau)=0$ occurs at some $\tau$ where $0 \leqslant \tau \leqslant \vartheta$, then we say that the differential game (1) can be completed within the time $\vartheta$.
Definition 5 A function $v(t, u), v:[0, T] \times l_{2} \rightarrow l_{2}$, that satisfies the conditions

(i) $v(t, u)=0,0 \leqslant t \leqslant \varepsilon$

(ii) $v(t, u)=u(t-\varepsilon), \varepsilon<t \leqslant T$,

where $\varepsilon$ is a positive number, is called a strategy of the evader.

Definition 6 A number $\vartheta$ is called optimal pursuit time if

(i) the differential game can be completed within the time $\vartheta$,

(ii) there exists a strategy $v(\cdot)$ of the evader such that $z(t) \neq 0,0 \leqslant t<\vartheta$, for any control $u(\cdot)$ of the pursuer. In this case we say that evasion is possible on $[0, \vartheta)$.

Problem. Find the optimal pursuit time in the differential game (1).

For $k=1,2, \ldots$, let

$$
A_{k}(t)=\left[\begin{array}{rr}
\mathrm{e}^{-\alpha_{k} t} \cos \beta_{k} t & -\mathrm{e}^{-\alpha_{k} t} \sin \beta_{k} t \\
\mathrm{e}^{-\alpha_{k} t} \sin \beta_{k} t & \mathrm{e}^{-\alpha_{k} t} \cos \beta_{k} t
\end{array}\right] .
$$

It is not difficult to show that the matrices $A_{k}(t)$ have the following properties:

$$
\begin{aligned}
A_{k}(t+h) & =A_{k}(t) A_{k}(h)=A_{k}(h) A_{k}(t), \\
\left|A_{k}(t) z_{k}\right| & =\left|A_{k}^{*}(t) z_{k}\right|=\mathrm{e}^{-\alpha_{k} t}\left|z_{k}\right|,
\end{aligned}
$$

where $A^{*}$ denotes the transpose of the matrix $A$.

\section{TIME-OPTIMAL CONTROL PROBLEM}

In this section, we find the minimum time required to steer the system from some initial point to the origin.

Let $C\left(0, T ; l_{2}\right)$ be the space of continuous functions $z(\cdot)$ such that $z(t) \in l_{2}$ for each $t, 0 \leqslant t \leqslant T$. The following proposition is true ${ }^{10}$.

Proposition 1 If $w(\cdot) \in S\left(\rho_{0}\right)$, and $\alpha_{k} \geqslant 0$, then for any given $T>0$ the following infinite system of differential equations

$$
\begin{array}{r}
\dot{x}_{k}=-\alpha_{k} x_{k}-\beta_{k} y_{k}+w_{1 k}, \\
\dot{y}_{k}=\beta_{k} x_{k}-\alpha_{k} y_{k}+w_{2 k}, \\
x_{k}(0)=x_{k 0}, \quad y_{k}(0)=y_{k 0},
\end{array}
$$

$k=1,2, \ldots$, has a unique solution $z(t)=$ $\left(z_{1}(t), z_{2}(t), \ldots\right), 0 \leqslant t \leqslant T$, in the space $C\left(0, T ; l_{2}\right)$. Of course, for $k=1,2, \ldots$,

$$
z_{k}(t)=A_{k}(t) z_{k 0}+\int_{0}^{t} A_{k}(t-s) w_{k}(s) \mathrm{d} s .
$$

It should be noted that this existence-uniqueness theorem for the system (3) was proved for any finite 
interval. Hence we consider the systems (1) and (3) on $[0, T]$, with $T$ being an arbitrary positive number.

For the system (3), first we consider the following time-optimal control problem ${ }^{2}$ :

$$
z(0)=z_{0}, z(\vartheta)=0, \vartheta \rightarrow \min _{w} .
$$

Let

$$
\varphi_{k}(t)= \begin{cases}\frac{\mathrm{e}^{2 \alpha_{k} t}-1}{2 \alpha_{k}}, & \alpha_{k}>0 \\ t, & \alpha_{k}=0, \quad t>0\end{cases}
$$

Since $1 / \varphi_{k}(t) \rightarrow+\infty$ as $t \rightarrow 0^{+}$for each $k$, the left-hand side of the equation

$$
\sum_{k=1}^{\infty} \frac{\left|z_{k 0}\right|^{2}}{\varphi_{k}(t)}=\rho_{0}^{2}
$$

approaches $+\infty$ as $t \rightarrow 0^{+}$. Moreover, the left part of (5) is a decreasing function of $t, t>0$, and

$$
\sum_{k=1}^{\infty} \frac{\left|z_{k 0}\right|^{2}}{\varphi_{k}(t)} \leqslant \frac{1}{t} \sum_{k=1}^{\infty}\left|z_{k 0}\right|^{2}=\frac{1}{t}\left\|z_{0}\right\|^{2} \rightarrow 0, \quad t \rightarrow \infty .
$$

Hence (5) has a unique root $t=\vartheta$.

Theorem 1 The number $\vartheta$ is the solution of the timeoptimal control problem (3)-(4).

Proof: A. Define the control

$$
w_{k}(t)=-\frac{1}{\varphi_{k}(\vartheta)} A_{k}^{*}(-t) z_{k 0}, \quad 0 \leqslant t \leqslant \vartheta .
$$

Then by (2)

$$
\begin{aligned}
& \sum_{k=1}^{\infty} \int_{0}^{\vartheta}\left|w_{k}(t)\right|^{2} \mathrm{~d} t \\
& \quad=\sum_{k=1}^{\infty} \frac{1}{\varphi_{k}^{2}(\vartheta)} \int_{0}^{\vartheta}\left|A_{k}^{*}(-t) z_{k 0}\right|^{2} \mathrm{~d} t \\
& \quad=\sum_{k=1}^{\infty} \frac{\left|z_{k 0}\right|^{2}}{\varphi_{k}^{2}(\vartheta)} \int_{0}^{\vartheta} \mathrm{e}^{2 \alpha_{k} t} \mathrm{~d} t=\sum_{k=1}^{\infty} \frac{\left|z_{k 0}\right|^{2}}{\varphi_{k}(\vartheta)}=\rho_{0}^{2} .
\end{aligned}
$$

Hence the control (6) is admissible.

B. Show that $z(\vartheta)=0$. Indeed,

$$
\begin{aligned}
\xi_{k}(\vartheta) & \doteq z_{k 0}+\int_{0}^{\vartheta} A_{k}(-s) w_{k}(s) \mathrm{d} s \\
& =z_{k 0}-\frac{1}{\varphi_{k}(\vartheta)} \int_{0}^{\vartheta} A_{k}(-s) A_{k}^{*}(-s) z_{k 0} \mathrm{~d} s \\
& =z_{k 0}-z_{k 0}=0, \quad k=1,2, \ldots
\end{aligned}
$$

Therefore $z_{k}(\vartheta)=A_{k}(\vartheta) \xi_{k}(\vartheta)=0, k=1,2, \ldots$.

C. We now show that $z(t) \neq 0,0 \leqslant t<\vartheta$, where $z(t)$ is the state of the system (3).

Assume the contrary. Then there exists an admissible control $w^{*}(\cdot)$ and time $\tau, 0 \leqslant \tau<\vartheta$, such that $z(\tau)=0$. Hence $\xi(\tau)=\left(\xi_{1}(\tau), \xi_{2}(\tau), \ldots\right)=0$, i.e.,

$$
\int_{0}^{\tau} A_{k}(-s) w_{k}^{*}(s) \mathrm{d} s=-z_{k 0} .
$$

We use the following proposition (see, for example, Ref. 12).

Proposition 2 Let $B(t), \quad 0 \leqslant t \leqslant \vartheta_{0}$, be a continuous matrix-function of the order $n$, and its determinant be not identically 0 on $\left[0, \vartheta_{0}\right]$. Then among the measurable functions $u(\cdot), u:\left[0, \vartheta_{0}\right] \rightarrow$ $R^{n}$, satisfying the condition

$$
\int_{0}^{\vartheta_{0}} B(s) u(s) \mathrm{d} s=z_{0}
$$

the control defined at almost everywhere on $\left[0, \vartheta_{0}\right]$ by the formula

$u(s)=B^{*}(s) F^{-1}\left(\vartheta_{0}\right) z_{0}, \quad F\left(\vartheta_{0}\right)=\int_{0}^{\vartheta_{0}} B(s) B^{*}(s) \mathrm{d} s$,

gives the minimum to the functional

$$
\int_{0}^{\vartheta_{0}}|u(s)|^{2} \mathrm{~d} s
$$

For the matrix

$$
F_{k}(\tau)=\int_{0}^{\tau} A_{k}(-s) A_{k}^{*}(-s) \mathrm{d} s
$$

we have $F_{k}^{-1}(\tau)=\left(1 / \varphi_{k}(\tau)\right) E_{2}$. Then by Proposition 2 the control

$$
\begin{aligned}
w_{0}(t) & =\left(w_{10}(t), w_{20}(t), \ldots\right), \\
w_{k 0}(t) & =-\frac{1}{\varphi_{k}(\tau)} A_{k}^{*}(-t) z_{k 0}, \quad k=1,2, \ldots,
\end{aligned}
$$

satisfies (7) and gives the minimum to the functional

$$
I(w(\cdot))=\sum_{k=1}^{\infty} \int_{0}^{\tau}\left|w_{k}(t)\right|^{2} \mathrm{~d} t .
$$

Substituting (8) into the functional $I$ gives

$$
\begin{aligned}
I\left(w^{*}(\cdot)\right) & \geqslant I\left(w_{0}(\cdot)\right)=\sum_{k=1}^{\infty} \frac{\left|z_{k 0}\right|^{2}}{\varphi_{k}(\tau)} \\
& >\sum_{k=1}^{\infty} \frac{\left|z_{k 0}\right|^{2}}{\varphi_{k}(\vartheta)}=\rho_{0}^{2} .
\end{aligned}
$$

This means the control (8) is not admissible. Then $w^{*}(\cdot)$ is not admissible. Contradiction. This completes the proof. 


\section{DIFFERENTIAL GAME PROBLEM}

We now consider the differential game (1). As shown in the previous section, the equation

$$
\sum_{k=1}^{\infty} \frac{\left|z_{k 0}\right|^{2}}{\varphi_{k}(t)}=(\rho-\sigma)^{2}
$$

has a unique solution $t=\vartheta_{1}$. We can assume, by choosing $T$ if necessary, that $\vartheta_{1}<T$.

Theorem $2 \vartheta_{1}$ is the optimal pursuit time in the game (1).

Proof: 1. First we show that the differential game (1) can be completed within the time $\vartheta_{1}$. To this end we offer to the pursuer the following strategy

$u_{k}(t, v)=v_{k}(t)-\frac{1}{\varphi_{k}\left(\vartheta_{1}\right)} A^{*}(-t) z_{k 0}, \quad k=1,2, \ldots$

Let $v(\cdot)$ be any admissible control of the evader. Admissibility of the strategy (9) can be shown by using the Minkowski inequality as follows:

$$
\begin{aligned}
\left(\sum_{k=1}^{\infty} \int_{0}^{\vartheta_{1}}\left|u_{k}(t, v(t))\right|^{2} \mathrm{~d} t\right)^{1 / 2} & \\
= & \left(\sum_{k=1}^{\infty} \int_{0}^{\vartheta_{1}}\left|v_{k}(t)-\frac{1}{\varphi_{k}\left(\vartheta_{1}\right)} A^{*}(-t) z_{k 0}\right|^{2} \mathrm{~d} t\right)^{1 / 2} \\
\leqslant & \left(\sum_{k=1}^{\infty} \int_{0}^{\vartheta_{1}}\left|v_{k}(t)\right|^{2} \mathrm{~d} t\right)^{1 / 2} \\
& +\left(\sum_{k=1}^{\infty} \int_{0}^{\vartheta_{1}} \frac{1}{\varphi_{k}^{2}\left(\vartheta_{1}\right)}\left|A^{*}(-t) z_{k 0}\right|^{2} \mathrm{~d} t\right)^{1 / 2} \\
\leqslant & +\rho-\sigma=\sigma .
\end{aligned}
$$

It is easy to show that $z_{k}\left(\vartheta_{1}\right)=0, k=1,2, \ldots$.

2. We now show that evasion is possible on the time interval $\left[0, \vartheta_{1}\right)$. The evader's strategy consists of two parts. If $\rho(t)>\sigma(t)$, where

$$
\begin{aligned}
\rho(t) & =\left(\rho^{2}-\int_{0}^{t}\|u(s)\|^{2} \mathrm{~d} s\right)^{1 / 2}, \\
\sigma(t) & =\left(\sigma^{2}-\int_{0}^{t}\|v(s)\|^{2} \mathrm{~d} s\right)^{1 / 2}, \\
\|u\|^{2} & =\sum_{k=1}^{\infty}\left|u_{k}\right|^{2}=\sum_{k=1}^{\infty}\left(u_{1 k}^{2}+u_{2 k}^{2}\right),
\end{aligned}
$$

we set

$$
v_{k}(t)=-\frac{1}{\varphi_{k}\left(\vartheta_{1}\right)} \frac{\sigma}{\rho-\sigma} A_{k}^{*}(-t) z_{k 0}, \quad k=1,2, \ldots
$$

If $\rho(t)=\sigma(t)$ at some $t \in\left[0, \vartheta_{1}\right)$, then the evader will use the second part of his strategy, which will be constructed later.

A. Show that if the evader uses the control (10), then $z(t) \neq 0, t \in\left[0, \vartheta_{1}\right)$, while $\rho(t) \geqslant \sigma(t)$. We assume the contrary, the game is completed at some time $t=\tau<\vartheta_{1}$, that is,

$$
z_{k}(\tau)=A_{k}(\tau) \eta_{k}(\tau)=0, k=1,2, \ldots,
$$

hence

$$
\begin{aligned}
\eta_{k}(\tau) \doteq z_{k 0} & -\int_{0}^{\tau} A_{k}(-s) u_{k}(s) \mathrm{d} s \\
& +\int_{0}^{\tau} A_{k}(-s) v_{k}(s) \mathrm{d} s=0, \quad k=1,2, \ldots
\end{aligned}
$$

when

$$
\rho(\tau) \geqslant \sigma(\tau) .
$$

From (10) we obtain

$$
\begin{aligned}
\int_{0}^{\tau} A_{k}(-s) & u_{k}(s) \mathrm{d} s \\
= & z_{k 0}+\int_{0}^{\tau} A_{k}(-s) v_{k}(s) \mathrm{d} s \\
& =z_{k 0}+\frac{\sigma}{\rho-\sigma} \frac{\varphi_{k}(\tau)}{\varphi_{k}\left(\vartheta_{1}\right)} z_{k 0}, \quad k=1,2, \ldots
\end{aligned}
$$

In accordance with Proposition 2 the minimum of the functional

$$
\int_{0}^{\tau}\left|u_{k}(s)\right|^{2} \mathrm{~d} s
$$

is attained at

$$
u_{k}(s)=\frac{1}{\varphi_{k}(\tau)} A_{k}^{*}(-s) z_{k 0}\left(1+\frac{\sigma}{\rho-\sigma} \frac{\varphi_{k}(\tau)}{\varphi_{k}\left(\vartheta_{1}\right)}\right)
$$

almost everywhere on $[0, \tau]$. So

$$
\begin{aligned}
& \int_{0}^{\tau}\left|u_{k}(s)\right|^{2} \mathrm{~d} s \\
& =\frac{1}{\varphi_{k}(\tau)}\left|z_{k 0}\right|^{2}\left(1+\frac{\sigma}{\rho-\sigma} \frac{\varphi_{k}(\tau)}{\varphi_{k}\left(\vartheta_{1}\right)}\right)^{2}, k=1,2, \ldots
\end{aligned}
$$

According to (10)

$\int_{0}^{\tau}\left|v_{k}(s)\right|^{2} \mathrm{~d} s=\frac{\sigma^{2}}{(\rho-\sigma)^{2}} \frac{\varphi_{k}(\tau)}{\varphi_{k}^{2}\left(\vartheta_{1}\right)}\left|z_{k 0}\right|^{2}, \quad k=1,2, \ldots$

Hence for $k=1,2, \ldots$,

$$
\begin{aligned}
\int_{0}^{\tau}\left|u_{k}(s)\right|^{2} \mathrm{~d} s & -\int_{0}^{\tau}\left|v_{k}(s)\right|^{2} \mathrm{~d} s \\
= & \frac{1}{\varphi_{k}(\tau)}\left|z_{k 0}\right|^{2}+2 \frac{\sigma}{\rho-\sigma} \frac{1}{\varphi_{k}\left(\vartheta_{1}\right)}\left|z_{k 0}\right|^{2} .
\end{aligned}
$$


Consequently,

$$
\begin{aligned}
\sum_{k=1}^{\infty} & \left(\int_{0}^{\tau}\left|u_{k}(s)\right|^{2} \mathrm{~d} s-\int_{0}^{\tau}\left|v_{k}(s)\right|^{2} \mathrm{~d} s\right) \\
& =\sum_{k=1}^{\infty} \frac{1}{\varphi_{k}(\tau)}\left|z_{k 0}\right|^{2}+\frac{2 \sigma}{\rho-\sigma} \sum_{k=1}^{\infty} \frac{1}{\varphi_{k}\left(\vartheta_{1}\right)}\left|z_{k 0}\right|^{2} .
\end{aligned}
$$

As $\tau<\vartheta_{1}$ and the function $\varphi_{k}(t), \quad t>0$, is increasing, then in agreement with the definition of $\vartheta_{1}$ we have

$$
\sum_{k=1}^{\infty} \frac{1}{\varphi(\tau)}\left|z_{k 0}\right|^{2}>\sum_{k=1}^{\infty} \frac{1}{\varphi\left(\vartheta_{1}\right)}\left|z_{k 0}\right|^{2}=(\rho-\sigma)^{2} .
$$

Hence by (12)

$$
\begin{aligned}
\sum_{k=1}^{\infty}\left(\int_{0}^{\tau}\left|u_{k}(s)\right|^{2} \mathrm{~d} s-\int_{0}^{\tau}\left|v_{k}(s)\right|^{2} \mathrm{~d} s\right) \\
>(\rho-\sigma)^{2}+2(\rho-\sigma)^{2} \frac{\sigma}{\rho-\sigma}=\rho^{2}-\sigma^{2} .
\end{aligned}
$$

Hence

$$
\sigma^{2}-\sum_{k=1}^{\infty} \int_{0}^{\tau}\left|v_{k}(s)\right|^{2} \mathrm{~d} s>\rho^{2}-\sum_{k=1}^{\infty} \int_{0}^{\tau}\left|u_{k}(s)\right|^{2} \mathrm{~d} s,
$$

that is, $\sigma^{2}(\tau)>\rho^{2}(\tau)$, which contradicts the assumption that $\rho(\tau) \geqslant \sigma(\tau)$ (see, (11)).

Thus if the evader uses the control (10), then $z(t) \neq 0, t \in\left[0, \vartheta_{1}\right)$ whenever $\rho(t) \geqslant \sigma(t)$.

B. If $\rho\left(t_{1}\right)<\sigma\left(t_{1}\right)$ at some time $t_{1} \in\left[0, \vartheta_{1}\right)$ then by continuity of the functions $\rho(t), \sigma(t), t \geqslant 0$, and the inequality $\rho(0)>\sigma(0)$ there exists $\tau, 0 \leqslant \tau<t_{1}$ to hold the equality $\rho(\tau)=\sigma(\tau)$. As proved in the above that $z(t) \neq 0,0 \leqslant t \leqslant \tau$, and hence $z(\tau) \neq 0$.

In its turn the inequality $z(\tau)=$ $\left(z_{1}(\tau), z_{2}(\tau), \ldots\right) \neq 0$, implies that $z_{k}(\tau)=$ $A_{k}(\tau) \eta_{k}(\tau) \neq 0$ for some $k$. Hence $\eta_{k}(\tau) \neq 0$. Consider the following two-dimensional system

$\eta_{k}(t)=\eta_{k}(\tau)-\int_{\tau}^{t} A_{k}(-s)\left(u_{k}(s)-v_{k}(s)\right) \mathrm{d} s, t \geqslant \tau$,

associated with the number $k$. Construct a strategy for the evader that ensures the inequality $z_{k}(t) \neq 0$, $\tau \leqslant t \leqslant T$. Set

$$
v_{k}(s)= \begin{cases}0, & \tau \leqslant s \leqslant \tau+\varepsilon, \\ u_{k}(s-\varepsilon), & \tau+\varepsilon<s \leqslant T .\end{cases}
$$

In the following the positive number $\varepsilon$ will be chosen. If $\tau \leqslant t \leqslant \tau+\varepsilon$, then

$$
\eta_{k}(t)=\eta_{k}(\tau)-\int_{\tau}^{t} A_{k}(-s) u_{k}(s) \mathrm{d} s
$$

and

$$
\begin{aligned}
\left|\eta_{k}(t)\right| & \geqslant\left|\eta_{k}(\tau)\right|-\left|\int_{\tau}^{t} A_{k}(-s) u_{k}(s) \mathrm{d} s\right| \\
& \geqslant\left|\eta_{k}(\tau)\right|-\int_{\tau}^{t}\left|A_{k}(-s) u_{k}(s)\right| \mathrm{d} s \\
& =\left|\eta_{k}(\tau)\right|-\int_{\tau}^{t} \mathrm{e}^{\alpha_{k} s}\left|u_{k}(s)\right| \mathrm{d} s \\
& \geqslant\left|\eta_{k}(\tau)\right|-\left(\int_{\tau}^{\tau+\varepsilon} \mathrm{e}^{2 \alpha_{k} s} \mathrm{~d} s \cdot \int_{\tau}^{\tau+\varepsilon}\left|u_{k}(s)\right|^{2} \mathrm{~d} s\right)^{1 / 2} .
\end{aligned}
$$

Since

$$
\int_{0}^{T}\left|u_{k}(s)\right|^{2} \mathrm{~d} s \leqslant \rho^{2},
$$

the right part of the last inequality approaches $\left|\eta_{k}(\tau)\right|$ as $\varepsilon \rightarrow 0$.

$$
\begin{aligned}
& \text { If } \tau+\varepsilon<t \leqslant T \text {, then we have } \\
& \begin{aligned}
\eta_{k}(t)=\eta_{k}(\tau)-\int_{\tau}^{t} A_{k}(-s) u_{k}(s) \mathrm{d} s \\
\quad+\int_{\tau+\varepsilon}^{t} A_{k}(-s) u_{k}(s-\varepsilon) \mathrm{d} s \\
=\eta_{k}(\tau)-\int_{\tau}^{t} A_{k}(-s) u_{k}(s) \mathrm{d} s \\
\quad+\int_{\tau}^{t-\varepsilon} A_{k}(-s-\varepsilon) u_{k}(s) \mathrm{d} s \\
=\eta_{k}(\tau)+\int_{\tau}^{t-\varepsilon}\left[A_{k}(-s-\varepsilon)-A(-s)\right] u_{k}(s) \mathrm{d} s \\
\quad+\int_{t-\varepsilon}^{t} A_{k}(-s) u_{k}(s) \mathrm{d} s .
\end{aligned}
\end{aligned}
$$

Using the Cauchy-Schwartz inequality yields

$$
\begin{aligned}
&\left|\eta_{k}(t)\right| \geqslant\left|\eta_{k}(\tau)\right|-a_{k} \cdot\left(\int_{\tau}^{t-\varepsilon}\left|u_{k}(s)\right|^{2} \mathrm{~d} s\right)^{1 / 2} \\
&-\left(\int_{t-\varepsilon}^{t} \mathrm{e}^{2 \alpha_{k} s} \mathrm{~d} s \cdot \int_{t-\varepsilon}^{t}\left|u_{k}(s)\right|^{2} \mathrm{~d} s\right)^{1 / 2}
\end{aligned}
$$

where

$$
a_{k}=\left(\int_{\tau}^{t-\varepsilon}\left\|A_{k}(-s-\varepsilon)-A_{k}(-s)\right\|^{2} \mathrm{~d} s\right)^{1 / 2} .
$$

It is clear that $\left|\eta_{k}(\tau)\right|>0$, and the second and the third terms of the right part of the last inequality approach 0 as $\varepsilon \rightarrow 0$.

Thus there exists $\varepsilon_{0}>0$ such that $\eta_{k}(t)>$ $\left|\eta_{k}(\tau)\right| / 2, t \geqslant \tau$, whenever $0<\varepsilon<\varepsilon_{0}$. Hence $z_{k}(t) \neq 0, \tau \leqslant t \leqslant T$. This implies $z(t) \neq 0$, $\tau \leqslant t \leqslant T$. The proof of the theorem is complete. 


\section{CONCLUSIONS}

In the present paper, we have studied optimal pursuit game problem for the infinite system of DE (1) in the Hilbert space $l_{2}$. Control functions are subjected to integral constraints. The main results of the paper are as follows. (i) We have solved a time optimal control problem and found an equation to find the optimal time, (5). Also we have constructed optimal control. (ii) We have studied optimal pursuit problem for the differential game with integral constraints. We have constructed optimal strategies for the players and given an equation to find optimal pursuit time.

Research on methods for solving control and/or differential game problems for systems described by infinite number of ODEs has very promising future since many control and/or differential game problems described by PDEs can be reduced to such systems (see, for example, Refs. 4-9).

Control problems for infinite systems of differential equations require new methods. Many methods of finite dimensional spaces don't work even for infinite systems of simple form. For example, consider the following controlled system described by the first order differential equations

$$
\dot{z}_{k}=-\lambda_{k} z_{k}+w_{k}, z_{k}(0)=z_{k 0}, k=1,2, \ldots
$$

where $z_{k}, z_{k 0}, w_{k} \in \mathbb{R}, z(t)=\left(z_{1}(t), z_{2}(t), \ldots\right) \in$ $l_{2}, t \geqslant 0 ; w_{k}, k=1,2, \ldots$, are control parameters, and $\lambda_{k}, 0<\lambda_{1} \leqslant \lambda_{2} \leqslant \cdots \rightarrow$ $\infty$. Admissible controls are defined as functions $w(t)=\left(w_{1}(t), w_{2}(t), \ldots\right)$ with measurable components $w_{i}(t)$ subjected to geometric constraint

$$
\sum_{k=1}^{\infty} w_{k}^{2}(t) \leqslant \rho^{2}, \quad t \geqslant 0,
$$

where $\rho>0$ is a given number. Such a constraint is obtained if we consider the constraint on the norm of the form $\|v(\cdot, t)\| \leqslant \rho$ (see (5) in Ref. 8) instead of the constraint (1.6) in Ref. 6. It is natural to investigate the following time optimal control problem for the system (13) in the Hilbert space $l_{2}$ :

$$
z_{k}(T)=0, k=1,2, \ldots, \quad T \rightarrow \min
$$

which is an open problem. This problem can also be extended to systems with arbitrary positive coefficients $\lambda_{k}$. Another open problem for simple motion differential games in the Hilbert space $l_{2}$ was formulated in the conclusion of Ref. 11.

Acknowledgements: This study was partially supported by the research grant (RUGS) of the Universiti Putra Malaysia, No. 05-02-12-1868RU.

\section{REFERENCES}

1. Isaacs R (1965) Differential Games, Wiley, New York.

2. Pontryagin LS, Boltyanskii VG, Gamkrelidze RV, Mishchenko YeE (1969) Mathematical Theory of Optimal Processes, Nauka, Moscow.

3. Il'in VA, Tikhomirov VV (1999) The wave equation with boundary control at two ends and the problem of complete damping of a vibration process. Differentsialnie Uravneniya 35, 692-704.

4. Butkovskiy AG (1975) Control Methods in Systems with Distributed Parameters, Nauka, Moscow.

5. Avdonin SA, Ivanov SA (1989) The Controllability of Systems with Distributed Parameters and Families of Exponentials, UMKVO, Kiev.

6. Chernous'ko FL (1992) Bounded controls in systems with distributed parameters. Prikl Mat Mekh 56, 810-26.

7. Tukhtasinov M (1995) On some problems in the theory of differential pursuit games in systems with distributed parameters. Prikl Mat Mekh 59, 979-84.

8. Satimov NYu Tukhtasinov M (2006) Game problems on a fixed interval in controlled first-order evolution equations. Math Notes 80, 578-89.

9. Ibragimov GI (2003) A problem of optimal pursuit in systems with distributed parameters. J Appl Math Mech 66, 719-24.

10. Ibragimov GI, Azamov A, Hasim RM (2008) Existence and uniqueness of the solution for an infinite system of differential equations. Journal KALAM, International Journal of Mathematics and Statistics 1, 9-14.

11. Ibragimov GI, Hasim RM (2010) An evasion differential game in Hilbert space. Int Game Theor Rev 12, 239-51.

12. Lee EB, Markus L (1967) Foundations of Optimal Control Theory, Wiley, New York. 